\title{
Moisture Effects on Gold Nanowear
}

Megan Pendergast ${ }^{1}$, Alex A. Volinsky ${ }^{1}$, Xiaolu Pang ${ }^{1,2}$, and Robert Shields ${ }^{1}$

${ }^{1}$ Department of Mechanical Engineering, University of South Florida, 4202 E. Fowler Ave. ENB118, Tampa, FL, 33620

${ }^{2}$ Department of Materials Physics and Chemistry, University of Science and Technology Beijing, Beijing, 100083, China, People's Republic of

\begin{abstract}
The addition of water results in the higher wear rate of gold compared to experiments performed in the ambient environment (approximately $60 \%$ humidity). This higher wear rate in water has been observed with the AFM, Hysitron Triboindenter, and additionally in single pass scratch tests performed with the Taber Shear/Scratch tester. These tests were preformed using silicon nitride cantilevers in the AFM and a diamond tip in Hysitron and in the Taber instrument. Tests performed in the ambient atmosphere resulted in slightly reduced surface roughness, while much higher wear rate was observed in water. Ambient scratch tests consistently produced slightly shallower scratch trenches than wet scratches as a function of increasing normal load. Single scan lines provide valuable information about the mechanisms and progression of the nanoscale wear. The different components of scratch friction are investigated to explore the main contributors to the nanoscale scratching of gold.
\end{abstract}

\section{INTRODUCTION}

Abrasive wear resistance generally correlates with a material's hardness, however, this correlation was found not to hold true for thin coatings [1]. Additionally, many common wheel-based wear testers are too harsh for coatings. Scratch testing can provide a better evaluation of material abrasive resistance that is deposited as a thin coating. In many small scale applications, thin gold films are used as interconnects for electrical devices, therefore a scratch test can be a valuable experiment in evaluating gold wear properties. Technology continually strives to achieve smaller products, which pushes to reduce coating thickness. However, these coatings are still expected to provide the same tribological performance [2]. Much of scratch testing is devoted to providing information about a coating's practical adhesion, but wear resistance is equally significant [3].

In terms of quantifying material's scratch resistance, several definitions have been proposed, such as dynamic hardness, tangential hardness, and specific grooving energy. Recently an ASTM scratch standard was produced; however problems still lie in the reproducibility and the terms definitions [4]. To apply the definitions for scratch hardness, most often the contact area needs to be calculated, a difficult task if working with a material that can recover a large percentage of deformation upon unloading [5].

A scratch test is most often performed with a diamond tip scratching along the surface with a constant normal load. The depth of scratch is then analyzed to provide information about the material's scratch resistance. Another method useful in very thin 
films is to constantly ramp up the normal load until breakdown of the coating occurs. This method is beneficial when instrument resolution becomes a factor in measuring scratches $5 \mathrm{~nm}$ or less in depth. When the coating is penetrated, that load is defined as the critical load [3, 6-7], which is determined through the frictional force. When the frictional force becomes discontinuous or irregular, it is attributed to coating breakdown. This method is fairly reliable, but more recent methods involve using a conductive carbide blade to measure the electrical contact resistance (ECR) at the cutting point. The critical load of the coating is obtained when the resistance value is zero if the tip scratches through the coating to a conductive material underneath it. Scratch testing with the ramped normal load method becomes complicated because the underlay or substrate can be a significant factor in the coating's scratch resistance. The deeper the scratch, the more substrate properties influence the scratch resistance. This composite interaction is always measured during scratch testing using ramp loading because the scratch goes to the point of breakdown, involving the underlay [8].

It is generally accepted that much of scratch frictional forces come from a combination of ploughing and adhesive components. For reciprocating scratch test, it has been shown that the plastic ploughing component wanes off, leaving the majority of frictional force due to adhesive friction until coating delamination [9].

Not only can coating adhesion and wear resistance be obtained from the scratch test, but information about the critical plastic strain resistance can also be gained [10]. It has been difficult to experimentally determine failure strain values, because the strain fields in during wear are different from those in conventional tensile, compressive and torsion tests. Using an acoustic emission (AE) sensor, the point of microfracture during the scratch can be obtained, correlating this moment to the maximum plastic strain the material can withstand.

\section{EXPERIMENTAL DETAILS}

A Tabor Shear/Scratch Tester was used in the following scratch experiments on gold films. The conical diamond tip was used for testing. This tip has a point diameter of $178 \mu \mathrm{m}$. The gold sample for testing was a $3 \mu \mathrm{m}$ sputtered gold on silicon, cleaned prior to experiments with ethanol.

As the machine operates similar to a record player, some modifications had to be made for experiments to be carried out on smaller samples. A standard CD fits into the sample holder and the gold sample was mounted onto a CD surface using cyanoacrylate (Superglue). To allow experiments to be performed in the presence of water, a plastic case was modified to fit on the scratch tester; equipped with a rubber gasket to prevent water leakage into the machine. With this contraption, the CD with sample applied can be set into the plastic case and water can be poured in without damaging the instrument.

Ten single scratch scans were performed on the sample, first five in the ambient conditions, and later five with the sample submerged in water. Normal load varying experiments were performed on the $3 \mu \mathrm{m}$ sputtered gold sample, with normal loads varying from 25 to $125 \mathrm{~g}$. A profilometer was used for analyzing the scratch tracks after the experiments.

Experiments were also performed on the smaller scale with an AFM using a silicon nitride tapping mode tip (50 $\mathrm{nm}$ tip radius). The higher spring constant of a 
tapping mode tip $(50 \mathrm{~N} / \mathrm{m})$ allowed wear experiments to be performed over $1 \mathrm{x} 1 \mu \mathrm{m}^{2}$ area with $2 \mu \mathrm{N}$ normal load.

\section{RESULTS}

Before the profilometer scans of the scratches were obtained, images of the wear tracks were obtained in an optical microscope. Figure 1a displays a scratch performed in the ambient air environment and Figure 1b displays two scratches performed with the sample submerged in water.
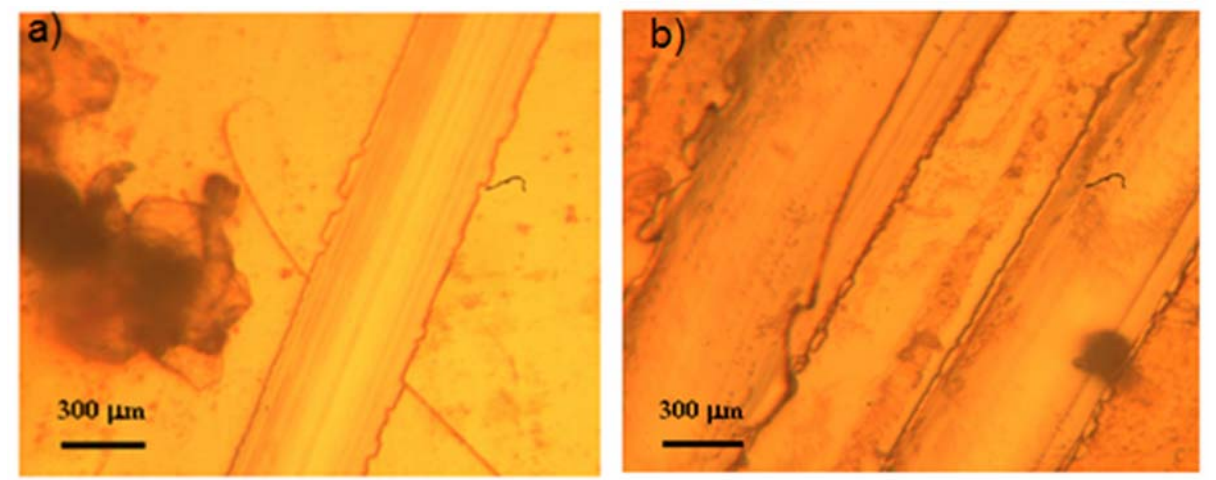

Figure 1. Optical micrographs of a) a scratch performed in ambient air conditions and b) two scratches performed submerged in water.
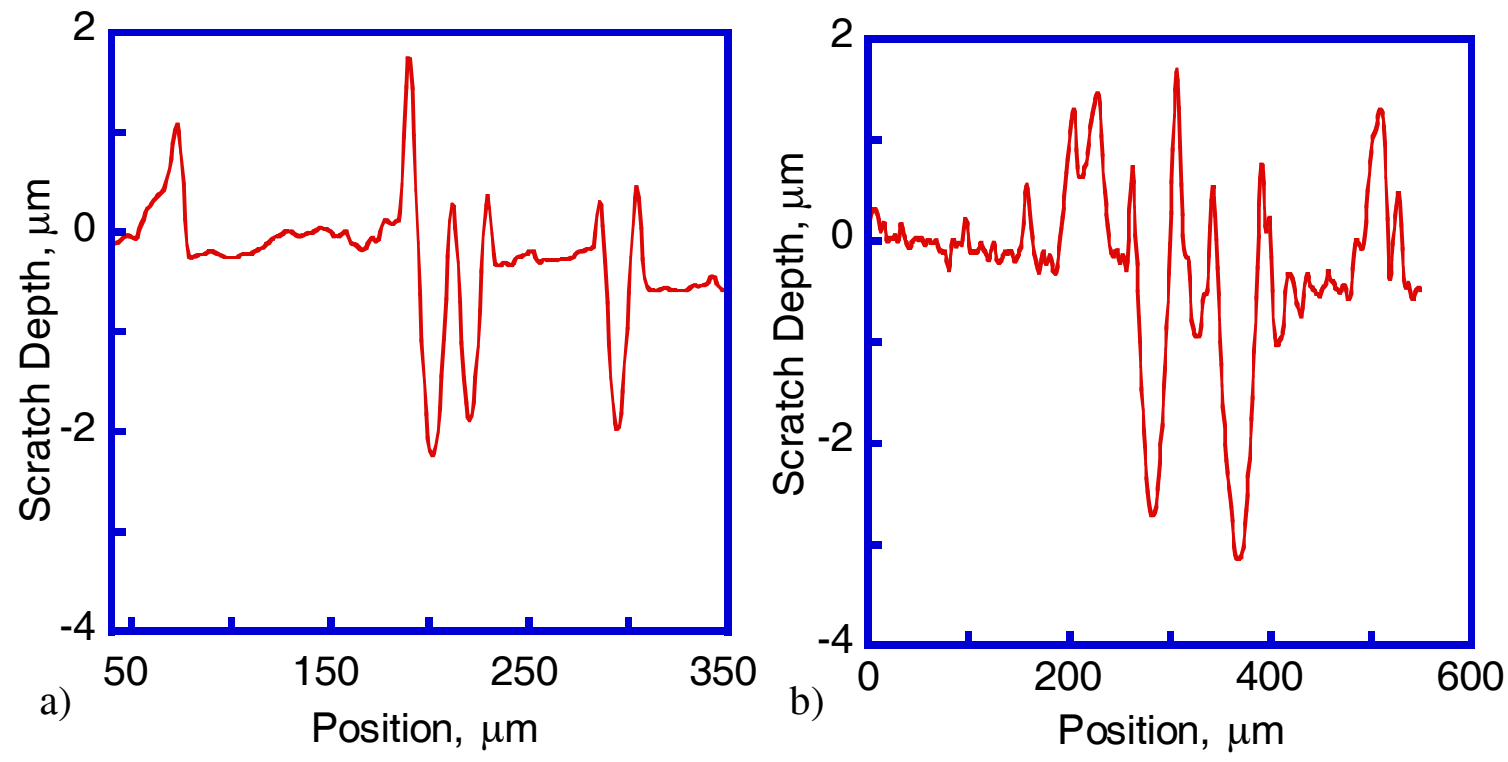

Figure 2. Depth profile of a) three scratches performed in ambient air conditions and b) two scratches performed in water.

The profilometer scratch depth profiles can be seen in Figures 2. The maximum depth of the ambient scratches reaches approximately $2 \mu \mathrm{m}$, and the maximum depth of the wet scratches is approximately $3 \mu \mathrm{m}$. 
Varying the normal load in both wet and ambient conditions produced the data shown in Figure 3. The depth of the wet scratches remains larger than the ambient, although the amount of difference varies.

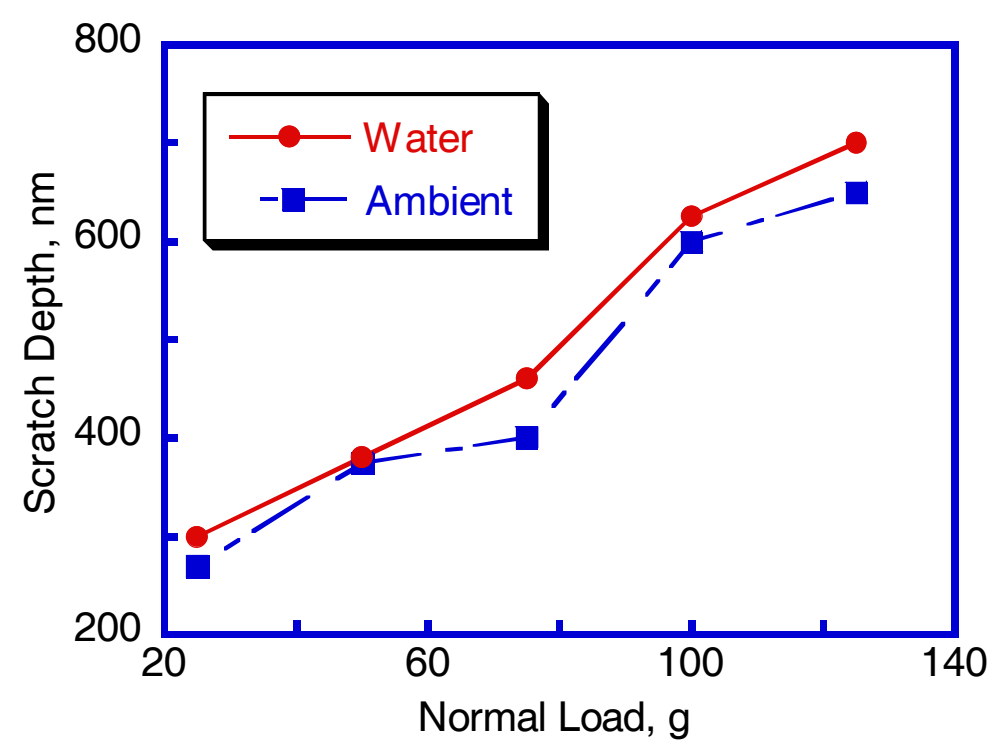

Figure 3. Scratch depth as a function of normal load for both wet and ambient (60\% humidity) conditions.

In order to gain an understanding of wear at the smaller scale, wear experiments were performed over a $1 \times 1 \mu \mathrm{m}^{2}$ square area in the AFM equipped with the fluid cell in wet and dry environments. Figure 4 displays the results of this testing, showing the average wear depth as a function of the number of scans.

It would be ideal to have more data and continue the test for a larger number of scans, but the water evaporated from the AFM fluid cell, only allowing 30 full scans. The addition of water to prolong this short scanning time caused the tip to lose contact with the sample due to the capillary forces of the added water. This loss of contact provided an offset and the test could not be continued in the exact same area.

\section{DISCUSSION}

As the tip moves across the surface of the gold surface, and the elastic limit is reached due to the contact pressure, material is plastically displaced. This material is pushed sideways and forms ridges on the sides of the scratch groove. This sideways movement of material is called ploughing or microploughing. The material removal mechanism for metals in the AFM has been proven to be mainly ploughing [11]. This is consistent with other experiments where low depth scratching friction coefficients are dominated by the ploughing term [1].

This implies that the role of adhesion is small in the shallow scratches. However, the addition of water to a scratch experiment has been shown to increase adhesion in the case of glass scratching [12]. There could be an increase in adhesion between the tip and 
the sample which causes an increase in static friction, explaining deeper scratch depths observed in wear areas of the gold film in the presence of water.

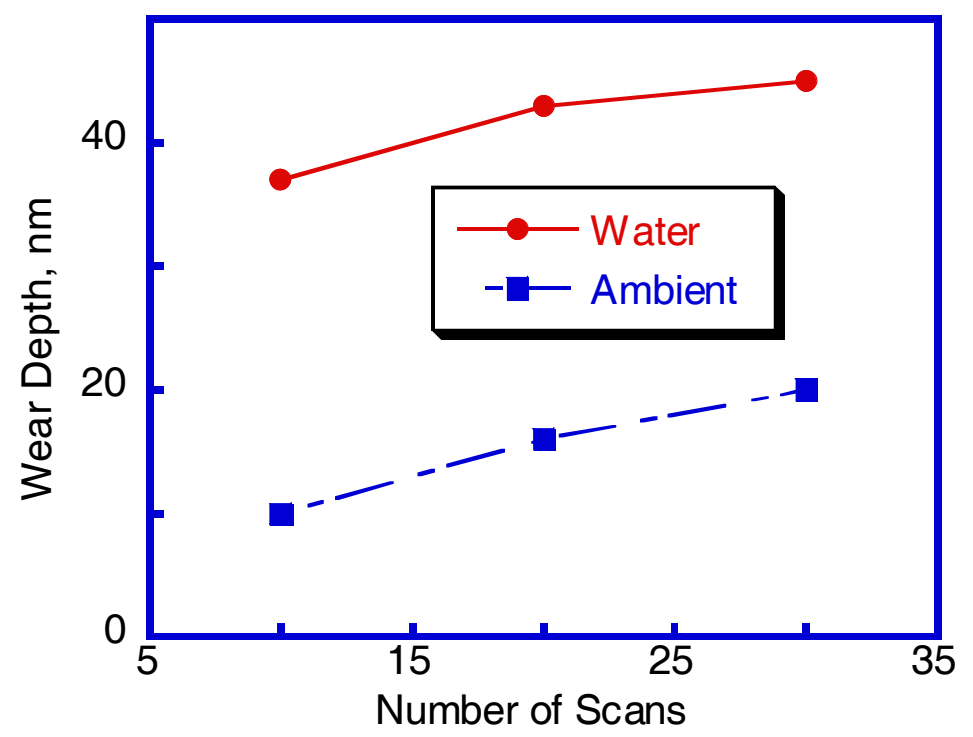

Figure 4. Results of the wear testing in ambient and wet conditions in the AFM system.

Additionally, capillary forces could be increasing the normal force in the AFM experiments, thus causing the increase in wear. Other experiments have shown the wear increase in water is due to a change in the wear mechanism, and not an increase in normal force. Experiments on epoxy/Kevlar pulp composites showed abrasive wear in wet conditions and mainly adhesion and plastic deformation in dry conditions [13]. In the progression of scratches and wear areas on gold, there are similarities in the dry and wet data in Figure 3, and it appears that the wet condition might be of the same wear mechanism, but at a higher normal force. The same trend is seen in the AFM wear depth in Figure 4.

\section{CONCLUSIONS}

The addition of water to gold wear experiments produced considerably deeper wear areas than its ambient counterpart. The same result was seen in scratch testing of gold, where scratches in water were deeper than the ambient scratches. Topography modification appears to be the main mechanism of ambient wear tests at the nanoscale, where much higher wear rate is observed in water.

\section{ACKNOWLEDGEMENTS}

This work is supported by the National Science Foundation (CMMI-0631526), the division of Design, Manufacture and Industrial Innovation, the Marie Curie Host Fellowship for the Transfer of Knowledge under the project "Nano-engineering for Expertise and Development - NEED" within the 6th EU Framework Program. We thank 
Hysitron, and Marek Szymonski's group at Jagiellonian University in Krakow, Poland for their collaboration.

\section{REFERENCES}

1. S.J. Bull, Wear 233-235, 412-423 (1999).

2. J. von Stebut, Surf. Coat. Tech. 200, 346-350 (2005).

3. A.P. Tschiptschin, C.M. Garzon, D.M. Lopez, Tribol. Int. 39, 167-174 (2006).

4. ASTM Standard G 171-03: Standard test method for scratch hardness of materials using a diamond stylus (2003).

5. Y. Xie, H.M. Hawthorne, Wear 240, 65-71 (2000).

6. M. Wong, G.T. Lim, A. Moyse, J.N. Reddy, H.J. Sue, Wear 256, 1214-1227 (2004).

7. M.H. Wong, Ph.D. Dissertation, Texas A\&M University (2003).

8. P. Bertrand-Lambotte, J.L. Loubet, C. Verpy, S. Pavan, Thin Solid Films 420-421, 281-286 (2002).

9. Q. Zhao, Z. Zhao, E. Kazazic, M. Embree, P. Trinh, T. Lam, S. Chang, Wear 252, 654-661 (2002).

10. J.C.M. Li, Mater. Sci. Eng. A 317, 197-203 (2001).

11. Z. Liu, J. Sun, W. Shen, Tribol. Int. 35, 511-522 (2002).

12. K. Li, Y. Shapiro, J.C.M. Li, Acta Mater. 46(15), 5569-5578 (1998).

13. J. Wu, X.H. Cheng, Wear 261, 1293-1297 (2006). 